\title{
MAGE-D4B is a novel marker of poor prognosis and potential therapeutic target involved in breast cancer tumorigenesis
}

\author{
Serena Germano ${ }^{1}$, Susan Kennedy ${ }^{2}$, Sweta Rani ${ }^{1}$, Grainne Gleeson ${ }^{2}$, Martin Clynes ${ }^{3}$, Padraig Doolan ${ }^{3}$, Susan McDonnell ${ }^{4}$, \\ Linda Hughes ${ }^{4}$, John Crown ${ }^{3}$ and Lorraine O'Driscoll ${ }^{1}$ \\ ${ }^{1}$ School of Pharmacy and Pharmaceutical Sciences, Trinity College Dublin, Dublin, Ireland \\ ${ }^{2}$ St. Vincent's University Hospital and Molecular Therapeutics for Cancer Ireland (MTCI), Dublin, Ireland \\ ${ }^{3} \mathrm{MTCl}, \mathrm{C} / \mathrm{o}$ National Institute for Cellular Biotechnology, Dublin City University, Dublin, Ireland \\ ${ }^{4}$ School of Chemical and Bioprocess Engineering, University College Dublin, Dublin, Ireland
}

\begin{abstract}
Melanoma-associated antigen (MAGE) family members are generally described as tumor-specific antigens. An association between MAGE-D4B and breast cancer has yet to be reported and the functional role of the encoded protein has never been established. We performed microarray analysis of 104 invasive breast tumors and matched non-cancerous breast biopsies. qPCR was used for validation in an independent biobank. To investigate the biological relevance of MAGE-D4B in breast tumorigenesis, its phenotypic effects were assessed in vitro. Overall, MAGE-D4B was detected in $43 \%$ of tumors while undetected in normal breast tissue. MAGE-D4B was found to correlate with tumor progression and to be an independent prognostic marker for poor outcome in term of relapse-free and overall survival, with potential predictive relevance in relation to response to chemotherapy. RNA interference-mediated knockdown of MAGE-D4B significantly hampered the invasive properties of Hs578T cells by affecting anchorage-independent growth, adhesion, migration and invasion affecting anchorageindependent growth, adhesion, migration and invasion and by modulating expression of invasion-suppressor gene E-cadherin.
\end{abstract}

Breast cancer is the most common cancer in women and one of the leading causes of death ${ }^{1}$ and it is characterized by high heterogeneity at the histopathologic and molecular levels, which is ultimately reflected in the clinical course of the dis-

Key words: MAGE-D4B, breast cancer, tumorigenesis, biomarker, therapeutic target

Abbreviations: ANOVA: analysis of variance; ECM: extracellular matrix; ER: oestrogen receptor; FBS: foetal bovine serum; MAGE: Melanoma-associated antigen; MHD: Mage Homology Domain; NFkB: nuclear factor kappa B; OS: overall survival; PBS: phosphate buffered saline; PCR: polymerase chain reaction; PR: progesterone receptor; qRT-PCR/qPCR: quantitative real-time polymerase chain reaction; RFS: relapse-free survival; STAT3: Signal transducer and activator of transcription 3; ATCC: American Tissue Culture Collection; CMF: cyclophosphamide, methotrexate, 5-fluorouracil Additional Supporting Information may be found in the online version of this article.

Grant sponsor: Health Research Board of Ireland; Grant number: RP/2006/77; Grant sponsor: Trinity College Dublin's Start-Up Funds for New Academics 2008/2009; Grant sponsor: Science Foundation Ireland's funding of Molecular Therapeutics for Cancer, Ireland; Grant number: 08/SRC/B1410; Grant sponsor: The Marie Keating Foundation

DOI: $10.1002 / \mathrm{ijc} .26200$

History: Received 27 Dec 2010; Accepted 2 May 2011; Online 26 May 2011

Correspondence to: Dr Lorraine O'Driscoll, School of Pharmacy and Pharmaceutical Sciences and MTCI, Trinity College Dublin, Dublin 2, Ireland, E-mail: lodrisc@tcd.ie ease and responses to treatment. The prognosis and clinical management of patients with breast cancer are commonly determined by clinicopathological factors and immunochemical markers. Histological type, grade, tumor size, lymph node involvement, estrogen (ER) and progesterone (PR) receptors status and HER2-receptor overexpression/gene amplification all influence prognosis and probability of response to systemic therapies; however, they fail to fully capture the varied clinical course of breast cancer. ${ }^{2}$

Expression of human melanoma-associated antigen $(M A G E)$ family genes has been recently associated with several types of cancer. ${ }^{3-7}$ The first human MAGE family member was discovered as a gene encoding a tumor-specific antigen. ${ }^{8}$ Since then, more than 50 MAGE genes have been identified and classified as type I and type II genes, based on differences in gene structure and tissue-specific gene expression. ${ }^{9}$ Type II MAGE-D genes are characterized by a more complex genomic structure compared to type I genes, with an open reading frame encoded by multiple exons. ${ }^{10}$ One of these genes, MAGE-D4B, has been reported in human malignancies, including gliomas, non-small-cell lung cancers and oral squamous cell carcinomas. ${ }^{11-13}$ However, an association of MAGE-D4B expression with breast cancer has not previously been established and the functional role of the encoded protein remains uncharacterized.

In this study, by genome-wide microarray analysis of a large cohort of patients, we identified MAGE-D4B to be differentially expressed in invasive breast tumors compared with normal breast tissue and to be significantly associated with poor outcome. Gene silencing experiments indicated that 
MAGE-D4B critically contributes to the tumorigenesis of breast cancer cells by regulating anchorage-independent growth, motility, invasiveness and expression of the invasionsuppressor gene E-cadherin. MAGE-D4B may thus represent a valuable prognostic biomarker for breast cancer and an attractive target for cancer therapy.

\section{Material and Methods \\ Patient characteristics and tissue sampling}

The study involved analysis of 104 cases of primary breast cancer from patients aged between 31 and 89 years at the time of diagnosis. Nineteen non-cancerous breast biopsies were included in the study. Tissue specimen were obtained from patients undergoing surgery at St Vincent's University Hospital (SVUH, Dublin, Ireland) and were analyzed following approval from SVUH Ethics Committee and with patients' informed consent. Patients were followed-up for a maximum of 3,026 days. Following surgical resection and pathologic evaluation, tissues were snap frozen in liquid nitrogen and stored at $-80^{\circ} \mathrm{C}$ before RNA isolation. Tumors were typed, graded and staging was performed as described previously. ${ }^{14}$

\section{RNA extraction, CDNA preparation and quantitative real- time PCR}

Total RNA was isolated from tissue samples using Tri reagent (Sigma-Aldrich, Arklow, Ireland) as described previously. ${ }^{15}$ RNeasy mini kit (Qiagen, Crawley, UK) was used for total RNA isolation from cell lines according to the manufacturer's instructions. cDNA was prepared from 100 ng RNA using MMLV reverse transcriptase (Sigma-Aldrich) according to the manufacturer's instructions. Real-time PCR was performed, using a Mastercycler ep realplex ${ }^{2} S$ system (Eppendorf, Cambridge, UK) and TaqMan Gene Expression Assays (Applied Biosystems, Warrington, UK). Samples were analyzed in triplicate, cDNA-absent and minus reverse transcriptase samples were included as negative controls. TaqMan Array Human Tumor Metastasis Fast 96-well plates (Applied Biosystems) were run on a $7900 \mathrm{HT}$ Fast Real-Time PCR System (Applied Biosystems) according to the manufacturer's instructions. Expression was normalized to the endogenous control gene GAPDH, identified as suitable based on our previous studies. ${ }^{16}$ The comparative $C_{\mathrm{T}}$ method was used for data analysis.

\section{Microarray}

A total of $100 \mathrm{ng}$ of RNA isolated from each breast tissue sample were amplified and labeled as described previously. ${ }^{14}$ Gene expression was examined using whole-genome microarrays (Affymetrix, U133 Plus 2.0) as reported previously. ${ }^{17}$

\section{Cell culture and transfection}

The Hs578Bst breast cell line was obtained from the American type culture collection (ATCC) and maintained according to ATCC guidelines. The Hs578T breast cancer cell line was obtained from ATCC and the highly invasive clone
Hs578Ts(i)8 was kindly provided by Susan McDonnell. ${ }^{18}$ These cells were grown in Dulbecco's Modified Eagle's Medium (Sigma-Aldrich) supplemented with 10\% fetal bovine serum (FBS, Lonza, Wokingham, UK), 5\% L-glutamine and $10 \mu \mathrm{g} / \mathrm{mL}$ bovine insulin (Sigma-Aldrich). Transfections of small interfering RNAs (siRNAs) and plasmid vectors were carried out with Lipofectamine 2000 (Invitrogen, Dun Laoghaire, Ireland) according to the manufacture's instructions. Transfected cells were harvested $48 \mathrm{hr}$ post-transfection for RNA extraction or biological assays and $72 \mathrm{hr}$ after transfection for protein extraction. siRNA were purchased from Ambion (Warrington, UK) and transfected at $50 \mathrm{nM}$. Two independent siRNAs were used to target MAGE-D4B (siRNA 1, pre-designed ID 130972; siRNA 2, 5' CCCGGAGCUCA GAGAGGAGtt $3^{\prime}$ ) and a scrambled-siRNA (Silencer Negative Control \#1) was used as a negative control. MAGE-D4B fulllength cDNA was subcloned from pOTB7 vector (clone ID3502168, Open Biosystems, Dublin, Ireland) by PCR into pcDNA 3.1(+) zeo vector (Invitrogen). The obtained construct was verified by DNA sequencing.

\section{Immunoblotting}

Total cellular proteins were extracted by using the SDS lysis buffer (250 mM Tris-HCl, pH 7.4, 2.5\% SDS), quantified with the micro BCA protein assay kit (Pierce, Dublin, Ireland) and dissolved in Laemmli sample buffer. Proteins were resolved by $10 \%$ SDS-PAGE and transferred to PVDF membranes (Millipore, Carrigtwohill, Ireland). Membranes were blocked in 5\% low-fat dry milk (Bio-Rad Laboratories, Blessington, Ireland) and incubated with the indicated primary antibodies (anti-MAGE-D4 clone H-188, Santa Cruz Biotechnology, Heidelberg, Germany; anti- $\beta$-tubulin, Sigma-Aldrich; anti-Stat3, anti-phospho Stat3, anti-NFkB and anti-phospho NFkB, Cell Signalling, Bray, Ireland). Membranes were incubated with appropriate horseradish peroxidase-conjugated secondary antibodies (Cell Signaling) and proteins were visualized by chemiluminescence (Millipore). Detection was performed with the Chemidoc exposure system (Bio-Rad Laboratories).

\section{Immunohistochemistry}

All steps were performed at room temperature unless otherwise indicated. Tissue sections were dewaxed in xylene $(2 \times$ $5 \mathrm{~min}$ ), rehydrated in grading alcohols $100 \%, 90 \%$ and $70 \%$ $(2 \times 3 \mathrm{~min}$ each) to $\mathrm{H} 2 \mathrm{O}$. Endogenous peroxidase activity was quenched by placing tissue sections in $3 \%(\mathrm{v} / \mathrm{v}) \mathrm{H}_{2} \mathrm{O}_{2}$ / distilled water for $15 \mathrm{~min}$. All slides were blocked for nonspecific staining with normal serum, (Vestastain ABC Kit, Vector Laboratories CA, PK-6101) for 20 min. Excess blocking serum was shaken off and $100 \mu \mathrm{L}$ of the primary antibody (MAGE D4) was added to each section. Specifically, anti-MAGE-D4 clone H-188 (Santa Cruz Biotechnology, Heidelberg, Germany) was diluted 1:500 in phosphate buffered saline (PBS; pH 7) and incubated overnight at $4^{\circ} \mathrm{C}$. Samples were then washed $(3 \times 5 \mathrm{~min})$ with $\mathrm{PBS}$, followed by a 30 
min. incubation with biotinylated secondary antibody (Vectastain ABC Kit, PK-6101). Finally, following another $3 \times 5$ min wash step, Vector DAB substrate for peroxidise (Vector Laboratories, SK-4700) was applied for $\sim 5 \mathrm{~min}$. All slides were washed $(3 \times 5 \mathrm{~min})$ in running water. Tissue sections were then lightly counter-stained with Harris Haematoxylin for $1 \mathrm{~min}$. After this, slides were dehydrated in grading alcohols $70 \%, 90 \%$ and $100 \%(2 \times 3 \mathrm{~min})$. Samples were then cleared in xylene and mounted in DPX (BDH, UK). Positive control using sections of glioma tissue (using same experimental conditions) and negative control samples (in which primary antibody were replaced with $100 \mu \mathrm{L}$ PBS) were included in all experiments.

\section{Cell proliferation assays}

Cells were plated in 96-well plates at a density of $5 \times 10^{3}$ cells/well and proliferation was measured at the indicated time points using the acid phosphatase assay. ${ }^{19}$

\section{Anchorage-independent growth assays}

Assays were performed using the CytoSelect ${ }^{\mathrm{TM}}$ 96-Well Cell Transformation kit (Cell Biolabs) according to the manufacturer's instructions. $7.5 \times 10^{3}$ cells/well were incubated for 8 days in a semisolid agar media before being lysed and detected with the CyQuant GR Dye in a fluorescence plate reader.

\section{Anoikis assays}

An amount of $1 \times 10^{5}$ cells/well were plated onto standard 24-well tissue plates or Poly(hydroxyethyl methacrylic) acidcoated (Sigma-Aldrich) plates and cultured for $24 \mathrm{hr} .100 \mu \mathrm{L}$ of Alamar blue dye (Serotec, Oxford, UK) were then added to each well and absorbance was measured at $570 \mathrm{~nm}$, with a reference wavelength of $600 \mathrm{~nm}$.

\section{Wound-healing assays}

An amount of $2 \times 10^{5}$ cells/well were seeded on 6-well plates, transfected and cultured for $48 \mathrm{hr}$ to confluency. Monolayers were scratched with a pipette tip and the resulting wounded areas were monitored by phase contrast microscopy and determined using NIH Image J software.

\section{Migration and invasion assays}

Assays were performed using $8 \mu \mathrm{m}$ pore size 24 -well Transwell chambers (BD Biosciences, Oxford, UK). For invasion assays the inserts were pre-coated with ECM (SigmaAldrich). An amount of $5 \times 10^{4}$ cells/well were seeded in the upper compartment and allowed to migrate for $48 \mathrm{hr}$. Cells in the upper chamber were mechanically removed and migrated cells were stained with crystal violet. Staining was solubilized in $10 \%$ acetic acid and absorbance was measured at $595 \mathrm{~nm}$.

\section{Adhesion assays}

Assays were performed on 24-well plates pre-coated with 25 $\mu \mathrm{g} / \mathrm{mL}$ Collagen IV solution (Sigma-Aldrich). To inhibit nonspecific binding, plates were then incubated with $1 \%$ bovine serum albumin (BSA) solution for $30 \mathrm{~min}$ at $37^{\circ} \mathrm{C}$. Cells were harvested and seeded in serum-free medium at $5 \times 10^{4}$ cells/well on pre-coated wells. After $30 \mathrm{~min}$ incubation at $37^{\circ} \mathrm{C}$, unattached cells were removed by washing with PBS and adherent cells were stained with crystal violet. Staining was solubilized in $10 \%$ acetic acid and absorbance was measured at $595 \mathrm{~nm}$.

\section{Immunofluorescence}

Cells were seeded onto glass coverslips and cultured for 24 hr. Cells were fixed with $3 \%$ paraformaldehyde solution, permeabilized with $0.4 \%$ Triton X-100 solution and stained with rabbit anti-MAGE-D4B (SantaCruz) and mouse anti-Paxillin (BD Biosciences) antibodies for $2 \mathrm{hr}$ at $37^{\circ} \mathrm{C}$. After extensive washes with $\mathrm{PBS}, 30 \mathrm{~min}$ incubation at $37^{\circ} \mathrm{C}$ with Alexa Fluor 544 anti-rabbit and 488 anti-mouse (Invitrogen) secondary antibodies, respectively, was performed. Coverslips were mounted with Pro-Long Antifade reagent containing DAPI (Invitrogen) and analyzed with an Olympus FV1000 Point-Scanning Confocal Microscope.

\section{ELISA}

PathScan Signaling Nodes Multi-Target Sandwich Elisa kit (Cell Signalling) was used according to the manufacturer's instructions. Data were normalized according to total protein content.

\section{Statistical analysis}

Statistical analyses of microarray results were performed using the SPSS 16.1 software package as described previously. ${ }^{14}$ Student's $t$-test or one-way ANOVA, followed by Bonferroni's post-test, were used to evaluate differences in gene expression, cell proliferation, migration and survival using Graph Pad Prism 5 (Graph Pad Software, La Jolla). A value of $p<0.05$ was considered statistically significant.

\section{Results}

MAGE-D4B is differentially expressed in breast tumors compared to normal tissues and is a prognostic marker of poor outcome

To identify mRNA transcripts differentially expressed in breast cancer with potential value as prognostic biomarkers, we performed a whole-genome microarray analysis of an extensive range of invasive breast tumors and non-cancerous breast biopsies. We found MAGE-D4B mRNA to be present in $43 \%(45 / 104)$ of the breast tumor specimens analyzed, while it was undetectable in all normal breast tissue samples $(p<0.0001)$. We then analyzed the relationship between MAGE-D4B expression and characteristics of the primary breast carcinomas. As detailed in Table 1, Chi-Square 
analysis indicated significant associations between MAGED4B mRNA expression and advanced tumor grade $(p=$ $0.045)$, ER negativity $(p=0.013)$ and Ki67 expression $(p=$ $0.004)$ in the tumor cells. MAGE-D4B mRNA expression also tended to be associated with PR negativity $(p=0.050)$ and with spread to lymph nodes $(p=0.074)$, although statistical significance was not reached. Interestingly, MAGE-D4B was also associated with relapse-free survival (RFS, $p=0.027$ ) and overall survival (OS, $p=0.008$ ) within 5 years of diagnosis. These data were further supported by Kaplan-Meier analysis, which indicated a significant unfavorable association between RFS ( $p=0.037)$ or OS $(p=0.013)$ and expression of MAGE-D4B [Fig. 1a(i,ii)]. Incidentally, to assess potential predictive relevance of MAGE-D4B, Kaplan-Meier analysis was independently performed on the sub-groups of cases where chemotherapy (in this case, CMF) was administered [Fig. $1 a$ (iii,iv)] or was not used [Fig. $1 a(\mathrm{v}, \mathrm{vi})]$, respectively. While the numbers in each sub-group were limited, this analysis suggest MAGE-D4B to be predictive of poor response to chemotherapy in terms of OS $(p=0.029)$. A similar association was also found for RFS, although statistical significance was not reached $(p=0.073)$.

Other factors investigated in this study by univariate analysis, which were found to be significantly associated with RFS and OS, include tumor size and grade, PR status, HER2 status, Ki67 expression and lymph node status (Supporting Information Table 1). To further validate the prognostic value of MAGE-D4B, multivariate analysis was performed and significant factors are summarized in Supporting Information Table 2. The Cox regression model indicated that expression of MAGE-D4B is an independent prognostic marker for RFS $(p=0.035)$ and OS $(p=0.019)$.

MAGE-D4B mRNA upregulation in tumors was further confirmed by qRT-PCR validation, performed on nine cancer biopsies and matching non-lesional breast tissues from the same patients. MAGE-D4B transcript was identified at various levels in eight tumor specimens, while its expression was below the detection sensitivity in all the normal matching samples (Fig. 1b). MAGE-D4B expression at protein level was also evaluated by immunohistochemical analysis on tumor and normal specimen, where possible. Consistently with the results obtained at the mRNA level, MAGE-D4B protein was undetectable in normal breast tissue, while displaying a marked staining in tumor tissues (representative images are shown in Supporting Information Fig. S1).

Taken together, these results indicate that MAGE-D4B expression is up-regulated in breast tumors and associates with reduced relapse-free survival and overall survival of breast cancer patients.

\section{MAGE-D4B expression correlates with invasiveness in syngenic breast cell lines}

The triple-negative Hs578T breast cancer cell line was selected as a suitable model for functional studies, given the availability of a syngenic non-tumorigenic cell line
Table 1. Correlation between clinicopathological factors and expression of MAGE-D4B mRNA in breast carcinomas

\begin{tabular}{lll}
\hline & \multicolumn{1}{c}{ MAGE-D4B } \\
\cline { 2 - 3 } Characteristics & $\begin{array}{l}\text { No. of positive/negative } \\
\text { cases (\% positivity) }\end{array}$ & $p$ \\
\hline
\end{tabular}

\begin{tabular}{|c|c|c|}
\hline \multicolumn{3}{|l|}{ Age (yr) } \\
\hline$<50$ & $12 / 27(44.4)$ & 0.886 \\
\hline$>50$ & $33 / 77$ (42.9) & \\
\hline \multicolumn{3}{|l|}{ Tumor size } \\
\hline $\mathrm{T} 1(<2 \mathrm{~cm})$ & $7 / 18(38.9)$ & 0.667 \\
\hline $\mathrm{T} 2(2-5 \mathrm{~cm})$ & $36 / 83(43.4)$ & \\
\hline T3 (>5cm) & $2 / 3(66.7)$ & \\
\hline \multicolumn{3}{|c|}{ Lymph node metastasis } \\
\hline Negative & $15 / 45(33.3)$ & 0.074 \\
\hline Positive & $30 / 59(50.8)$ & \\
\hline
\end{tabular}

Histology grade

$\begin{array}{lll}\text { I and II } & 17 / 51(33.3) & 0.045^{*} \\ \text { III } & 28 / 40(70)\end{array}$

$\begin{array}{lcc}\text { Histology type } & & \\ \text { IDC } & 39 / 82(47.6) & 0.088 \\ \text { ILC } & 6 / 17(35.3) & \\ \text { Special } & 0 / 5(0) & \\ \text { ER status } & & 0.013^{\star} \\ \text { Negative } & 21 / 34(61.8) & \\ \text { Positive } & 24 / 67(35.8) & \end{array}$

PR status

$\begin{array}{lll}\text { Negative } & 22 / 39(56.4) & 0.050\end{array}$

Positive 11/33 (33.3)

\begin{tabular}{lll} 
HER2 status & & \\
\hline Negative & $29 / 68(42.6)$ & 0.330 \\
\hline Positive & $13 / 24(54.2)$ & \\
\hline
\end{tabular}

Ki67

\begin{tabular}{lrr} 
Negative & $16 / 46(34.8)$ & $0.004^{*}$ \\
Positive & $20 / 29(68.9)$ & \\
RFS (relapsed $\leq 5$ yr of diagnosis) & $0.027^{*}$ \\
No & $18 / 54(33.3)$ & \\
Yes & $23 / 41(56.1)$ & $0.008^{*}$ \\
OS (died $\leq 5$ yr of diagnosis) & \\
No & $21 / 64(32.8)$ & \\
Yes & $18 / 29(62.1)$ & \\
\hline
\end{tabular}

$p$ values are from $X^{2}$ analyses.

${ }^{*}$ indicates significant parameter.

Abbreviations: IDC: invasive ductal carcinoma; ILC: invasive lobular carcinoma.

(Hs578Bst), established from normal tissue peripheral to the tumor, ${ }^{20}$ and a highly invasive (Hs578Ts(i)8) isogenic subclone. ${ }^{18}$ We evaluated MAGE-D4B mRNA levels by qRT-PCR in these cell lines. Interestingly, MAGE-D4B 

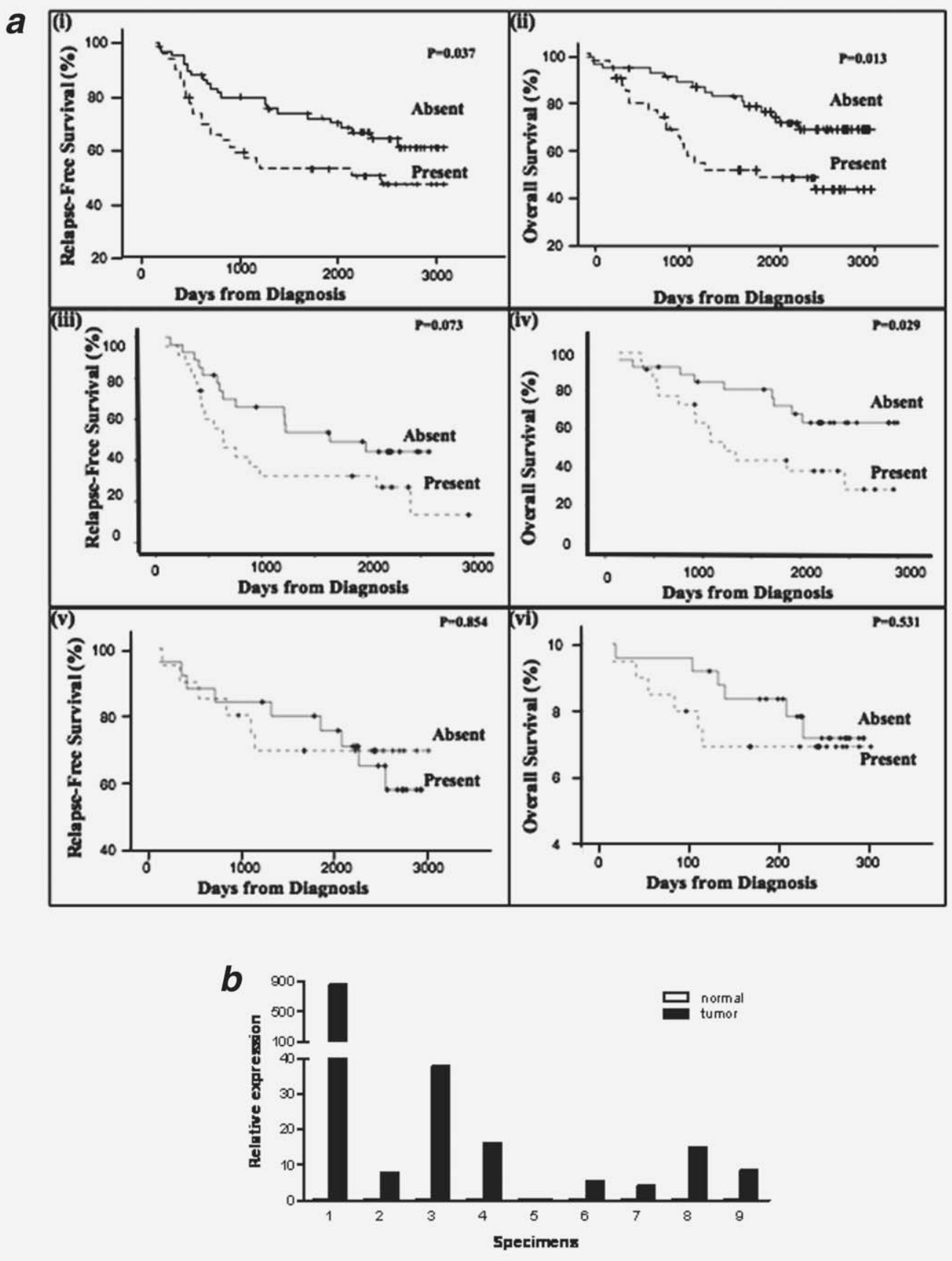

Figure 1. MAGE-D4B is expressed in human breast tumors and is associated with reduced relapse-free and overall survival. (a) Kaplan-Meier survival curves for MAGE-D4 presence or absence indicating a significant unfavorable association with (i) disease/relapse-free survival (RFS) and (ii) overall survival (OS). For patients who received adjuvant chemotherapy, while MAGE-D4 expression was not significantly associated with (iii) RFS, its presence was significantly associated with shortened (iv) overall survival times. This association was not found $[(\mathrm{v})$ and (vi)] for those who did not receive adjuvant chemotherapy. (b) qRT-PCR analysis showing MAGE-D4B mRNA to be absent from normal breast specimens and generally present in matching breast tumor specimens. Data are expressed as relative to a calibrator sample obtained by pooling equal amounts of all specimens. 
a

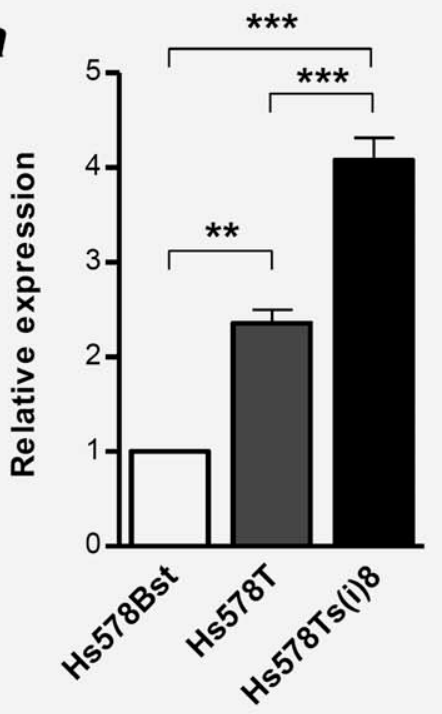

b

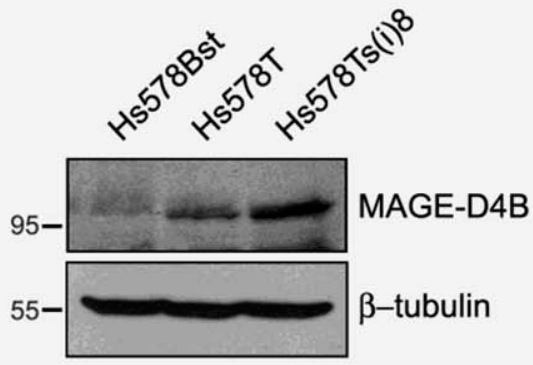

d

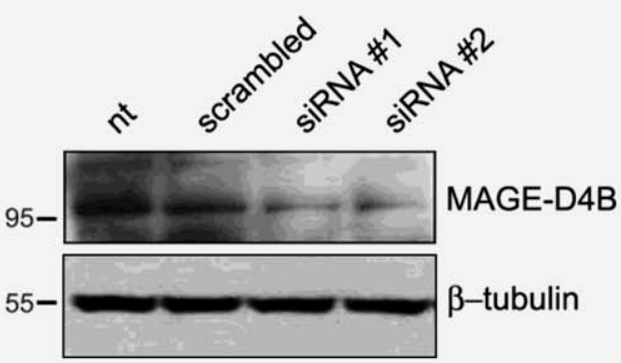

b

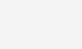

c

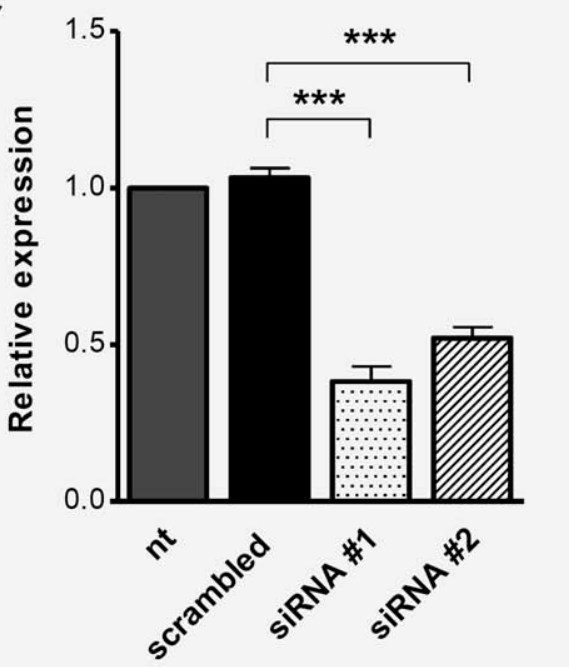




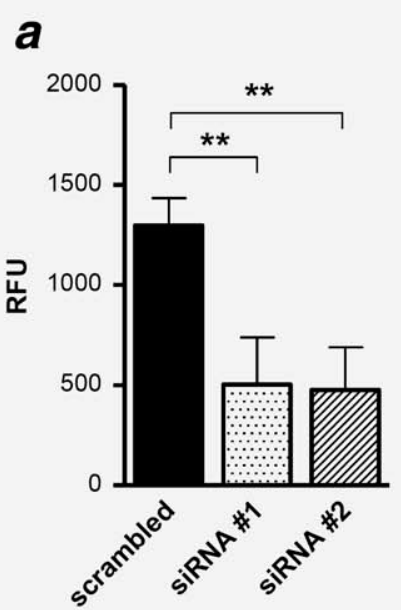

b

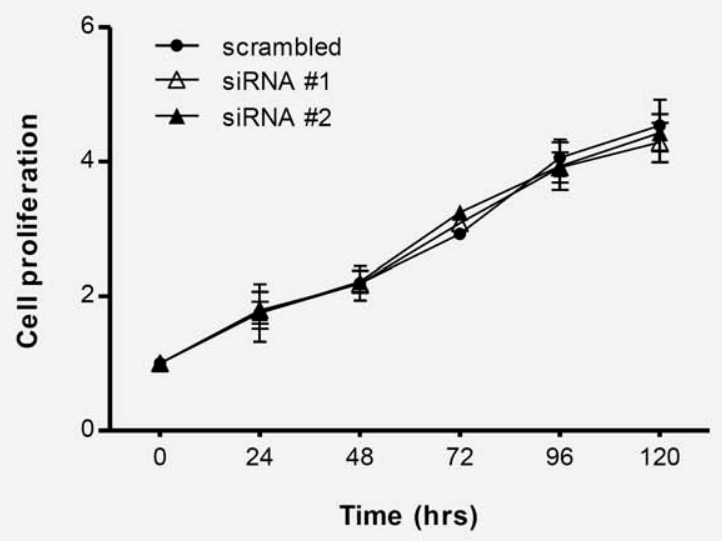

C

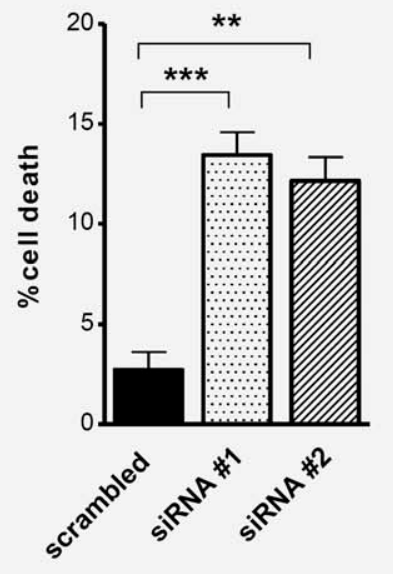

Figure 3. MAGE-D4B gene silencing affects anchorage-independent cell growth and survival. (a) Soft agar assays showing a marked reduction in colony formation upon MAGE-D4B-targeted siRNAs transfection of Hs578T cells. Relative fluorescence unit (RFU) is expressed as mean \pm SE of three independent experiments. (b) Proliferation assays on siRNA-transfected Hs578T cells indicating that monolayer cell growth was not affected by MAGE-D4B gene silencing. Cell proliferation is expressed as mean \pm SE of three independent experiments, relative to absorbance of seeded cells. (c) Anoikis assays were performed by culturing Hs578T cells on poly-HEMA coated dishes or uncoated control dishes. The level of anoikis was assessed as the percentage cell death relative to adherent controls, and expressed as mean $\pm \mathrm{SE}$ of three independent experiments. MAGE-D4B gene silencing resulted in a significant increase of cell death under anoikis conditions. ${ }^{\star \star} p<0.01 ;{ }^{* \star} p<0.001$.

\section{MAGE-D4B influences anchorage-independent growth and survival of breast cancer cells}

To determine whether MAGE-D4B could affect the mitogenic activity and metastatic potential of breast cancer cells, we examined the effects of MAGE-D4B gene silencing on anchorage-independent growth of Hs578T cells. Colony formation in soft-agar was dramatically reduced in MAGE-D4B siRNA-targeted cells compared to control cells (Fig. 3a). However, monolayer cell growth was not affected by MAGED4B gene knockdown. Proliferation assays indeed showed a comparable growth rate in cells transfected with MAGE-D4B specific siRNAs relative to control cells (Fig. 3b). Therefore, MAGE-D4B-mediated effects on soft-agar growth of Hs578T cells cannot be attributed to enhanced cell proliferation. To determine whether the reduced anchorage-independent growth observed upon MAGE-D4B gene silencing was related to a greater sensitivity to anoikis, we examined cell survival in conditions of prevented adhesion. ${ }^{21}$ Transfection of MAGE-D4B-targeted siRNAs resulted in increased cell death under anoikis conditions compared to control cells (Fig. 3c). Thus, MAGE-D4B plays a role in maintenance of tumorigenic potential of Hs578T breast cancer cells, most likely by regulating detachment-induced apoptosis.

\section{MAGE-D4B mediates control of invasive properties of breast cancer cells \\ We then examined whether MAGE-D4B expression is required for maintenance of the motile and invasive pheno- type in breast cancer cells. Wound-healing assays were used}

to model the effects of MAGE-D4B gene silencing on Hs578T cell motility. While the area of the wound was significantly recovered within $24 \mathrm{hr}$ by the migrating control cells transfected with scrambled siRNA, only a partial closure of the wound was observed in cells transfected with either of the MAGE-D4B specific siRNAs (Fig. 4a). The influence of MAGE-D4B knockdown on cellular motility and invasive capacity of Hs578T cells was independently assessed by using Transwell assays. MAGE-D4B siRNA-targeted cells displayed decreased motility, as their migration through $8.0 \mu \mathrm{m}$ pore size membranes was significantly reduced when compared to control cells (Fig. 4b). Comparable results were obtained when migration across a basement membrane-like layer was measured, indicating a role for MAGE-D4B in the regulation of breast cancer cells invasiveness (Fig. 4c). Notably, when MAGE-D4B overexpression in Hs578T cells was carried out, we observed a significant increase in both cell migration and invasion (Supporting Information Fig. S2). Together, these data suggest MAGE-D4B to be a critical regulator of invasive properties of breast cancer cells.

\section{MAGE-D4B gene silencing promotes adhesion of breast cancer cells and enhances E-cadherin expression}

To further confirm the involvement of MAGE-D4B in the invasive phenotype of breast cancer, cell adhesion assays were carried out. When plated on a collagen IV substrate, Hs578T cells transfected with MAGE-D4B siRNAs showed a significant increase of adhesion compared to control cells (Fig. 5a), indicating that MAGE-D4B expression may favor 


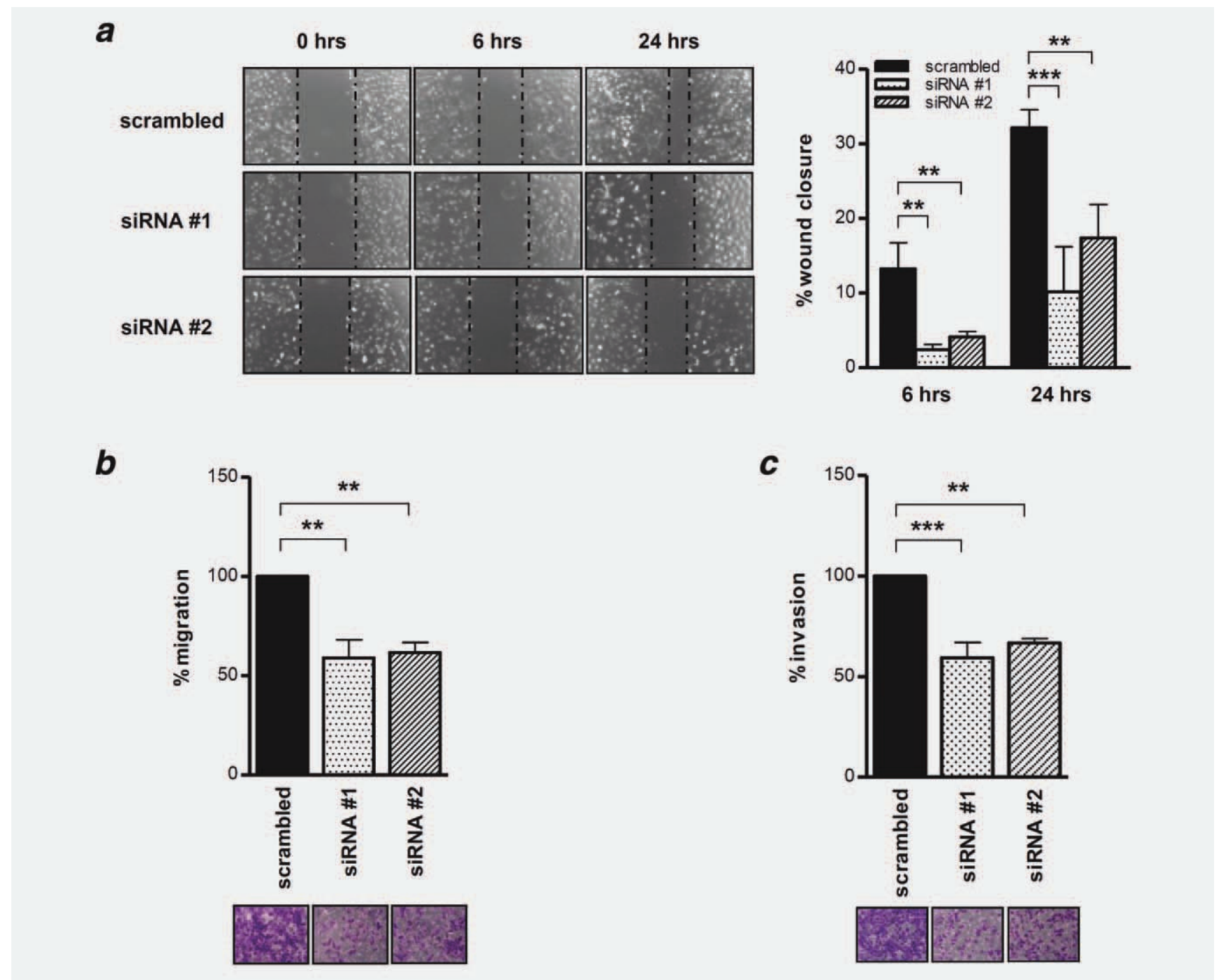

Figure 4. MAGE-D4B gene silencing hampers Hs578T cells motility and invasiveness. (a) The healing of wounds by migrated Hs578T cells 0,6 and $24 \mathrm{hr}$ after scratching of confluent monolayers was imaged (left; visual field at $\times 4$ ). Image analysis of four different wounds was performed and percentage of repaired wound \pm SE relative to time 0 is plotted (right). Cells transfected with MAGE-D4B-targeted siRNAs displayed reduced motility compared to that of scrambled-transfected control cells. (b) Migration assays were performed in Transwell chambers and migrated Hs578T cells to the undersurface were stained with crystal violet and imaged (representative images are shown in lower panel, visual field at $\times 10$ ). After solubilization of the dye, migration was expressed as percentage of the absorbance relative to scrambled-transfected cells (upper panel; mean \pm SE of four independent experiments). (c) Invasion assays were performed as in $b$, using Transwell chambers coated with ECM basement membrane. The assays showed a significant reduction of migration and invasion of Hs578T cells transfected with MAGE-D4B-targeted siRNAs when compared to scrambled-transfected cells. ${ }^{* \star} p<0.01$; ${ }^{\star * *} p<0.001$. [Color figure can be viewed in the online issue, which is available at wileyonlinelibrary.com.]

cell detachment and spreading. The focal adhesion-associated protein paxillin has been described as an important regulator of cell adhesion dynamics. ${ }^{22}$ We therefore analyzed the effects of MAGE-D4B silencing on paxillin immunostaining in Hs578T cells by confocal microscopy. In scrambled-transfected cells MAGE-D4B was predominantly distributed in the perinuclear region, while its signal was strongly reduced in cells transfected with either MAGE-D4B-targeted siRNAs, confirming the specificity of the detection (Fig. 5b). Noticeable changes in cell morphology were observed in MAGE-D4B
siRNAs-transfected cells; these cells appeared more rounded and displayed fewer protrusions than control cells. Interestingly, focal adhesion distribution and morphology appeared to be altered by MAGE-D4B gene silencing, as seen by an increase in the number of paxillin-positive focal adhesions, which were longer and appeared clumpy when compared to control cells (Fig. 5b). This effect on paxillin distribution was confirmed by more simply staining for paxillin following MAGE-D4B siRNA transfection (Fig. 5c, example results showing scrambled and siRNA \#2 effects). In an attempt to 


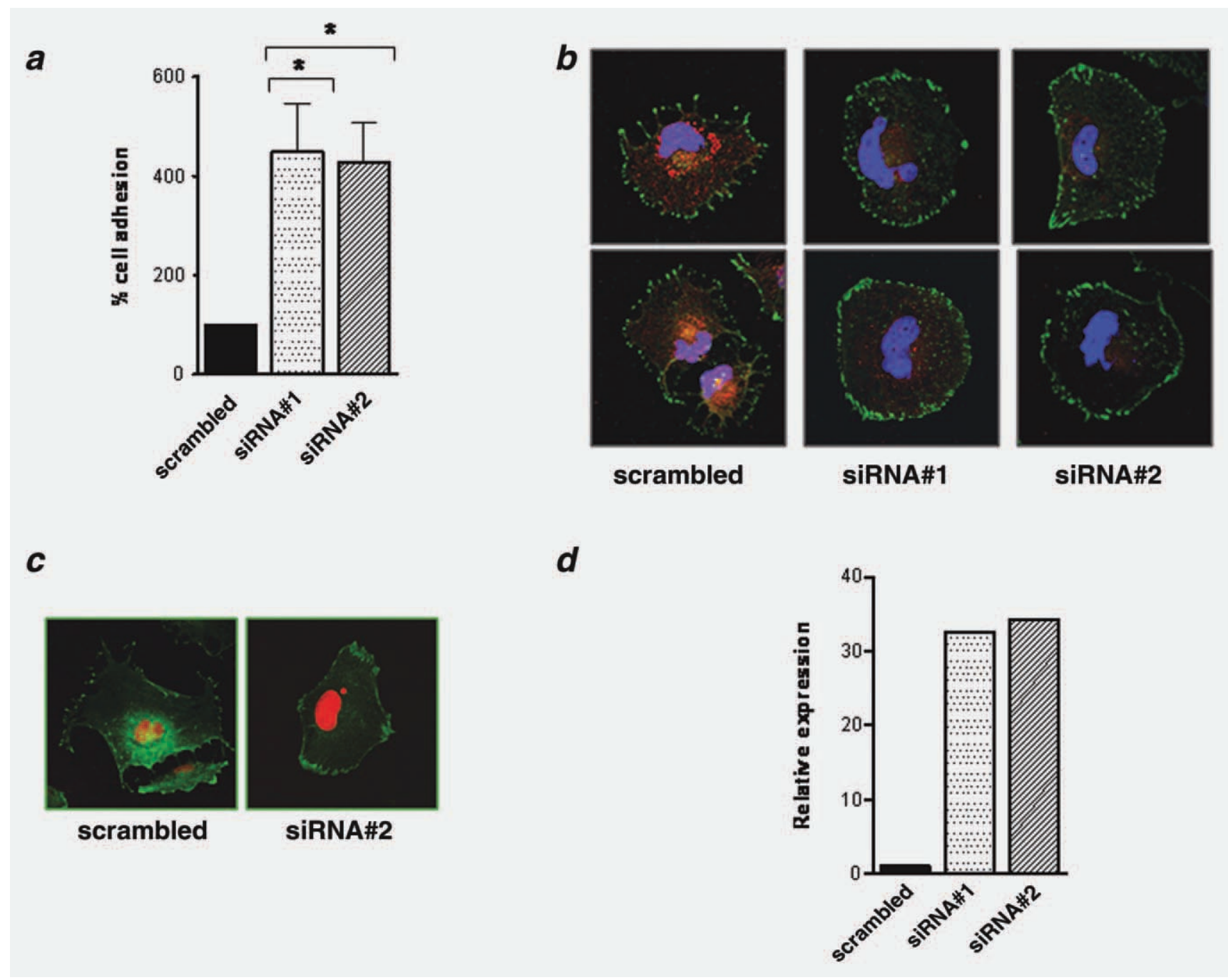

Figure 5. MAGE-D4B gene silencing increases adhesion and E-cadherin expression in Hs578T cells. (a) Adhesion assays, performed on collagen IV matrices, showed a significantly higher percentage of adhering cells upon MAGE-D4B-targeted siRNAs transfection. (b) Immunofluorescence analysis of Hs578T cells exhibiting merged signals of MAGE-D4B (red), paxillin (green) and DAPI (blue) nuclear counterstain (two representative fields are shown, visual field at $\times 60$ ). Cells transfected with either of the MAGE-D4B siRNAs displayed weaker MAGE-D4B signals and an increased number and size of paxillin-positive focal adhesions. (c) This effect of re-localization of paxillin following MAGE-D4B siRNA transfection was confirmed by staining for paxillin (green) only (DAPI red here) (scrambled and MAGE-D4B siRNA \#2 shown as example results, visual field at $\times 60$ ). (d) TaqMan Array analysis of metastases-related genes revealed marked upregulation of E-cadherin expression in Hs578T transfected with either of the MAGE-D4B specific siRNAs.

identify the molecular mechanisms underlying MAGE-D4B tumorigenic activity, a screening by qRT-PCR of the expression levels of 93 metastases-related genes was performed. Noteworthy, analysis of the results revealed a significant upregulation of the adhesion molecule E-cadherin, which was the only gene reaching a 5-fold modulation threshold following siRNA MAGE-D4B transfection. Indeed, a more than 30-fold increase of E-cadherin mRNA level was observed when MAGE-D4B-silenced cells were compared to control cells (Fig. $5 d$ ). These results clearly indicate that MAGE-D4B plays a role in invasiveness also by inhibition of cell adhesion, involving perturbation of focal adhesion and negative modulation of the invasion-suppressor gene E-cadherin.

\section{Discussion}

Recent microarray-based expression profiling studies have shed light on the molecular heterogeneity of breast cancer, which can be considered as a collection of molecularly distinct neoplastic diseases. ${ }^{23}$ Currently, diagnosis is mainly based on clinical and histopathological features and immunochemical markers. However, relatively few markers have established prognostic power to date. Therefore, there is a recurrent drive to identify biomarkers that will aid in its early diagnosis, prognosing outcome and predicting response to therapy, ultimately facilitating personalized therapies.

The results obtained by microarray analysis on the large cohort of patients tested in our study, revealed that MAGE- 
$\mathrm{D} 4 \mathrm{~B}$ is a valuable candidate as a novel biomarker in breast cancer. MAGE family genes were initially identified as genes encoding tumor-specific antigens, and since then MAGE type I genes have been extensively studied as attractive targets for anti-tumor immunotherapy. ${ }^{24,25}$ While expression of MAGE type I genes is restricted to a small number of normal tissues and specific stages of the embryonal development, MAGE type II genes are widely expressed in normal adult tissues. ${ }^{9,10}$ MAGE-D4B gene (previously known as MAGE-E1 and MAGE-D4) is a type II MAGE gene characterized by a unique distribution within normal tissues -being specifically expressed in brain and ovary- and with high expression levels documented in a variety of malignancies. ${ }^{11-13}$ Our whole-genome microarray analysis indicated MAGE-D4B transcript to be undetectable in normal mammary tissue and to be present in a large proportion (43\%) of breast cancer specimens. This result adds the mammary tissue to the list of adult tissues lacking MAGE-D4B and enlarges the spectrum of cancers where it has been found aberrantly expressed. The qRT-PCR analysis of a cohort of matched normal and tumor specimen from the same patients, further confirmed that the activation of MAGE-D4B expression is restricted to lesional tissues. Our study assigns for the first time a prognostic value to MAGE-D4B expression in breast cancer and our follow-up analysis demonstrated that MAGE-D4B expression is significantly associated with poor outcome in term of either overall survival or relapse-free survival. This is in accordance with the observation that MAGE-D4B positivity in our cohort is associated with known indicators of bad prognosis, such as high Ki67 expression, advanced tumor stage and ER negativity; which is in line with previous findings in lung cancer. ${ }^{26}$ Nevertheless, multivariate analysis demonstrated that MAGE$\mathrm{D} 4 \mathrm{~B}$ is an independent predictor of poor outcome, thus strongly supporting its value as a novel prognostic marker in breast cancer.

Incidentally, while the numbers of cases were more limited when sub-grouped into those who did and did not received adjuvant chemotherapy, the results of this analysis suggest the presence of MAGE-D4B to be predictive of poorer response to chemotherapy.

It is reasonable to speculate that among all cancer biomarkers, some can play a functional role in tumor biology, while the deregulated expression of others can merely be an epiphenomenon of the malignant transformation, with poor or no contribution to tumor formation and progression. Thus, the selection and characterization of the molecular mechanisms that play a key functional role in tumor behavior may contribute toward identifying novel targeted therapeutic approaches. ${ }^{27-29}$ The investigation of the functional relevance of MAGE-D4B expression was of particular relevance, since the biologic activities of this protein were yet to be elucidated. Searching for a suitable cellular model to perform an in vitro investigation of MAGE-D4B effects, we performed a preliminary qRT-PCR analysis of 10 breast cancer cell lines and found detectable levels of MAGE-D4B transcript in 50\% (not shown), in accordance with the in vivo results on tumor specimens. The selection of the triplenegative Hs578T cell line allowed us to observe an increase in MAGE-D4B expression levels moving from a syngenic non-tumorigenic cell line to a highly invasive isogenic subclone. This finding suggested a possible role of MAGE-D4B in the tumorigenic and invasive potential of Hs578T cells, which was then confirmed by loss-of- and gain-of-function experiments. Indeed, the effects of MAGE-D4B on migration, adhesion, invasion and anchorage-independent growth suggest a functional role for this protein in breast cancer tumorigenesis, at least in aggressive triple-negative cancer cells. In our patient cohort, it was not possible to verify an association of MAGE-D4B expression with triple-negativity, likely as a consequence of the limited number of triple-negative cases $(n=11)$. Nevertheless, $55 \%$ of triple-negative tumors expressed MAGE-D4B, suggesting that it could be a potential candidate for the management of this subset, where the lack of molecular therapeutic targets is limiting. The effects of MAGE-D4B on cell motility, adhesion and anoikis are in accordance with the observation that MAGE$\mathrm{D} 4 \mathrm{~B}$ gene silencing induces an increase in the number of focal adhesions and a redistribution of paxillin staining. Indeed, focal adhesions play a critical role in the regulation of cell migration and are also important sites of signal transduction. $^{30}$

The results here reported, which demonstrate for the first time a biological relevance of MAGE-D4B protein, are in contrast with that observed for MAGE-D1, which has been shown to promote cell cycle arrest and to impair migration and invasion of breast cancer cells. ${ }^{31,32}$ The different behavior of the two MAGE family members is in agreement with gene expression data, which indicate a reduced expression of MAGE-D1 in $38 \%$ of breast carcinoma cell lines, ${ }^{31}$ as opposed to the upregulation of MAGE-D4B in the tumor specimens observed in our study. Despite the presence of the Mage homology domain (MHD) in both proteins, MAGE-D1 and MAGE-D4B significantly differ at their amino-terminal portions, possibly accounting for their opposite activities. Noteworthy, it has been proposed, based on interaction studies, that functional antagonism among MAGE family proteins may exist. ${ }^{33}$ Future studies are needed to address the molecular mechanisms underlying MAGE-D4B biological activities. Our results indicate that a contribution to MAGE-D4B tumorigenic potential could be credited to the downregulation of the tumor suppressor E-cadherin, which plays a crucial role in the maintenance of cell-cell adhesion. Loss of E-cadherin expression has been described in several tumors, and has been shown to correlate with poor prognosis in breast cancer patients. ${ }^{34,35}$ The effect of MAGE-D4B on E-cadherin expression is currently under investigation and our preliminary results indicate that the inhibition of the activities (i.e., reduced phosphorylation) of transcription factors STAT3 and NFkB may be involved (see Supporting Information Fig. 3). 
In conclusion, while analysis of larger numbers of normal specimens would be required before diagnostic relevance could be claimed for MAGE-D4B and to assess the true likelihood that a selective cancer therapy could be developed based on interdiction of MAGE-D4B, the novel data presented here indicate MAGE-D4B as a new prognostic biomarker in breast cancer, whose importance can be greatly enhanced by future prospective longitudinal studies analyzing its potential role as a marker predictive of response to therapy. Furthermore, these studies suggesting that MAGE-D4B may be predictive of poor response to adjuvant chemotherapy should be advanced in larger studies and looking at responsiveness to other forms of chemotherapy as well as newer, more targeted, therapies. The presence of other MAGE type 2 proteins in normal tissues should be considered with particular regards when designing anti-MAGE-D4B therapies. In conclusion, our results-which we believe for the first time-assign a functional role to MAGE-D4B, may form the basis of future studies aimed at the characterization of MAGE-D4B as a target of therapeutic intervention.

\section{References}

1. Coughlin SS, Ekwueme DU. Breast cancer as a global health concern. Cancer Epidemiol 2009;33:315-8.

2. Rakha EA, Reis-Filho JS, Ellis IO. Combinatorial biomarker expression in breast cancer. Breast Cancer Res Treat 2010;120:293-308.

3. Atanackovic D, Hildebrandt Y, Jadczak A, Cao Y, Luetkens T, Meyer S, Kobold S, Bartels K, Pabst C, Lajmi N, Gordic M, Stahl $\mathrm{T}$, et al. Cancer-testis antigens MAGE-C1/CT7 and MAGE-A3 promote the survival of multiple myeloma cells. Haematologica 2010;95: 785-793.

4. Bergeron A, Picard V, LaRue H, Harel F, Hovington H, Lacombe L, Fradet Y. High frequency of MAGE-A4 and MAGE-A9 expression in high-risk bladder cancer. Int J Cancer 2009;125:1365-71.

5. Karpf AR, Bai S, James SR, Mohler JL, Wilson EM. Increased expression of androgen receptor coregulator MAGE-11 in prostate cancer by DNA hypomethylation and cyclic AMP. Mol Cancer Res 2009;7:523-35.

6. Pastorcic-Grgic M, Sarcevic B, Dosen D, Juretic A, Spagnoli GC, Grgic M. Prognostic value of MAGE-A and NYESO-1 expression in pharyngeal cancer. Head Neck 2010;32:1178-84.

7. Ries J, Mollaoglu N, Toyoshima T, Vairaktaris E, Neukam FW, Ponader S, Nkenke E. A novel multiple-marker method for the early diagnosis of oral squamous cell carcinoma. Dis Markers 2009;27:75-84.

8. van der Bruggen $\mathrm{P}$, Traversari C, Chomez P, Lurquin C, De Plaen E, Van den Eynde BJ, Knuth A, Boon T. A gene encoding an antigen recognized by cytolytic $\mathrm{T}$ lymphocytes on a human melanoma. Science 1991;254:1643-7.

9. Barker PA, Salehi A. The MAGE proteins: emerging roles in cell cycle progression, apoptosis, and neurogenetic disease. J Neurosci Res 2002;67:705-12.

10. Chomez P, De Backer O, Bertrand M, De Plaen E, Boon T, Lucas S. An overview of the MAGE gene family with the identification of all human members of the family. Cancer Res 2001;61:5544-51.

11. Sasaki M, Nakahira K, Kawano Y, Katakura H, Yoshimine T, Shimizu K, Kim SU, Ikenaka K. MAGE-E1, a new member of the melanoma-associated antigen gene family and its expression in human glioma. Cancer Res 2001;61:4809-14.

12. Tsai JR, Chong IW, Chen YH, Yang MJ, Sheu CC, Chang HC, Hwang JJ, Hung JY, Lin SR. Differential expression profile of MAGE family in non-small-cell lung cancer. Lung Cancer 2007;56:185-92.

13. Cheong SC, Chandramouli GV, Saleh A, Zain RB, Lau SH, Sivakumaren S, Pathmanathan R, Prime SS, Teo SH, Patel $\mathrm{V}$, Gutkind JS. Gene expression in human oral squamous cell carcinoma is influenced by risk factor exposure. Oral Oncol 2009; 45:712-9.

14. Martinez V, Kennedy S, Doolan P, Gammell P, Joyce H, Kenny E, Prakash Mehta J, Ryan E, O'Connor R, Crown J, Clynes M, O’Driscoll L. Drug metabolismrelated genes as potential biomarkers: analysis of expression in normal and tumour breast tissue. Breast Cancer Res Treat 2008;110:521-30.

15. Doolan P, Clynes M, Kennedy S, Mehta JP, Germano S, Ehrhardt C, Crown J, O'Driscoll L. TMEM25, REPS2 and Meis 1: favourable prognostic and predictive biomarkers for breast cancer. Tumour Biol 2009;30:200-9.

16. Kennedy S, Clynes M, Doolan P, Mehta JP, Rani S, Crown J, O’Driscoll L. SNIP/ p140Cap mRNA expression is an unfavourable prognostic factor in breast cancer and is not expressed in normal breast tissue. Br J Cancer 2008;98: 1641-5.

17. O’Driscoll L, McMorrow J, Doolan P, McKiernan E, Mehta JP, Ryan E, Gammell P, Joyce H, O’Donovan N, Walsh N, Clynes M. Investigation of the molecular profile of basal cell carcinoma using whole genome microarrays. Mol Cancer 2006;5: 74.

18. Hughes L, Malone C, Chumsri S, Burger AM, McDonnell S. Characterisation of breast cancer cell lines and establishment of a novel isogenic subclone to study migration, invasion and tumourigenicity. Clin Exp Metastasis 2008;25:549-57.

19. O’Driscoll L, Linehan R, Liang YH, Joyce H, Oglesby I, Clynes M. Galectin-3 expression alters adhesion, motility and invasion in a lung cell line (DLKP), in vitro. Anticancer Res 2002;22:3117-25.

20. Hackett AJ, Smith HS, Springer EL, Owens RB, Nelson-Rees WA, Riggs JL, Gardner MB. Two syngeneic cell lines from human breast tissue: the aneuploid mammary epithelial (Hs578T) and the diploid myoepithelial (Hs578Bst) cell lines. J Natl Cancer Inst 1977;58:1795-1806.

21. Folkman J, Moscona A. Role of cell shape in growth control. Nature 1978;273: 345-9.

22. Deakin NO, Turner CE. Paxillin comes of age. J Cell Sci 2008;121:2435-44.

23. Sotiriou C, Pusztai L. Gene-expression signatures in breast cancer. $N$ Engl J Med 2009;360:790-800.

24. Caballero OL, Chen YT. Cancer/testis (CT) antigens: potential targets for immunotherapy. Cancer Sci 2009;100: 2014-21.

25. Liu BB, Ye SL, He P, Liu YK, Tang ZY. MAGE-1 and related MAGE gene expression may be associated with hepatocellular carcinoma. J Cancer Res Clin Oncol 1999;125:685-9.

26. Ito S, Kawano Y, Katakura H, Takenaka K, Adachi M, Sasaki M, Shimizu K, Ikenaka K, Wada H, Tanaka F. Expression of MAGE-D4, a novel MAGE family antigen, is correlated with tumor-cell proliferation of non-small cell lung cancer. Lung Cancer 2006;51:79-88.

27. Brenton JD, Carey LA, Ahmed AA, Caldas C. Molecular classification and molecular forecasting of breast cancer: ready for clinical application? J Clin Oncol 2005;23: 7350-60.

28. Clynes M, O'Connor R, O’Driscoll L, Daly $\mathrm{C}$, Meleady P. Challenges in molecular analysis for individualized cancer therapy. Drug Discov Today 2003;8: 531-32. 
29. Di Cosimo S, Baselga J. Management of breast cancer with targeted agents: importance of heterogenicity. Nat Rev Clin Oncol 2010;7:139-47.

30. Turner CE. Paxillin interactions. J Cell Sci 2000;113:4139-40.

31. Tian XX, Rai D, Li J, Zou C, Bai Y, Wazer D, Band V, Gao Q. BRCA2 suppresses cell proliferation via stabilizing MAGE-D1. Cancer Res 2005;65:4747-53.
32. Du Q, Zhang Y, Tian XX, Li Y, Fang WG. MAGE-D1 inhibits proliferation, migration and invasion of human breast cancer cells. Oncol Rep 2009;22:659-65.

33. Sasaki A, Hinck L, Watanabe K. RumMAGE-D the members: structure and function of a new adaptor family of MAGE-D proteins. J Recept Signal Transduct Res 2005;25: 181-98.
34. Baranwal S, Alahari SK. Molecular mechanisms controlling E-cadherin expression in breast cancer. Biochem Biophys Res Commun 2009;384:6-11.

35. Berx G, Van Roy F. The E-cadherin/ catenin complex: an important gatekeeper in breast cancer tumorigenesis and malignant progression. Breast Cancer Res 2001;3:289-93. 